\title{
Correction: Kaiso is required for MTG16-dependent effects on colitis-associated carcinoma
}

\author{
Sarah P. Short ${ }^{1,2} \cdot$ Caitlyn W. Barrett ${ }^{1,2} \cdot$ Kristy R. Stengel $^{3} \cdot$ Frank L. Revetta $^{4} \cdot$ Yash A. Choksi $^{1,2,5}$. \\ Lori A. Coburn ${ }^{1,5,6} \cdot$ Mary K. Lintel $^{1}$ - Elizabeth M. McDonough ${ }^{1,7} \cdot$ M. Kay Washington ${ }^{4} \cdot$ Keith T. Wilson $\mathbb{1}^{1,2,4,5,6,8}$. \\ Egor Prokhortchouk ${ }^{9} \cdot \mathrm{Xi}_{\text {Chen }}{ }^{10} \cdot$ Scott W. Hiebert ${ }^{3,8} \cdot$ Albert B. Reynolds $^{2,8} \cdot$ Christopher S. Williams $^{1,2,5,6,8}$
}

Published online: 8 May 2019

๑) Springer Nature Limited 2019

\section{Correction to: Oncogene}

https://doi.org/10.1038/s41388-019-0777-7

published online 11 March 2019

In the original version of this article the authors noted that the GEO accession number for the relevant dataset was listed incorrectly as GSE12454.
Christopher S. Williams

Christopher.williams@vanderbilt.edu

1 Department of Medicine, Division of Gastroenterology, Vanderbilt University Medical Center, Nashville, TN 37232, USA

2 Program in Cancer Biology, Vanderbilt University, Nashville, TN 37232, USA

3 Department of Biochemistry, Vanderbilt University, Nashville, TN 37232, USA

4 Department of Pathology, Microbiology, and Immunology, Vanderbilt University Medical Center, Nashville, TN 37232, USA

5 Veterans Affairs Tennessee Valley Health Care System,
The correct number is instead GSE124544.

This has now been corrected in both the PDF and HTML versions of the Article.

Nashville, TN 37232, USA

6 Center for Mucosal Inflammation and Cancer, Vanderbilt University Medical Center, Nashville, TN 37232, USA

7 Department of Pediatrics, Division of Gastroenterology, Our Lady of the Lake Children's Hospital, Baton Rouge, TN 70808, USA

8 Vanderbilt Ingram Cancer Center, Nashville, TN 37232, USA

9 Institute of Bioengineering, Research Center of Biotechnology of the Russian Academy of Sciences, Moscow, Russia

10 Department of Public Health Sciences and the Sylvester Comprehensive Cancer Center, University of Miami Miller School of Medicine, Miami, FL, USA 